\title{
In-situ S/TEM Probing of the Coupling among Electrochemical, Thermal, and Mechanical Effect in Rechargeable Batteries
}

\author{
Chongmin Wang \\ Pacific Northwest National Laboratory, Richland, WA, USA.
}

Electrochemical driven functioning of battery inevitably induces thermal and mechanical effects, which in turn couple closely with the electrochemical process and collectively govern the performance of the battery. However, such a coupling effect has never been explicitly explored. We use in-situ TEM to demonstrate such a coupling effect. We discover that supplying a thermal perturbation to the delithiated $\mathrm{LiNi}_{0.6} \mathrm{Mn}_{0.2} \mathrm{Co}_{0.2} \mathrm{O}_{2}$ (NMC622) will lead to the nucleation and propagation of intragranular cracks in the lattice, which shows dependence on the fraction of Li that has been extracted from the lattice and will not happen for the case of pristine or with Li been re-inserted sample. Comprehensive mechanics analyses revealed that thermal stress associated with electrochemically induced phase inhomogeneity and internal pressure due to oxygen gas evolution are the two primary driving forces for nucleation and propagation intergranular crack, resembling a "popcorn" fracture mechanism. The present work unveils that the electrochemical process and its trigged thermal and mechanical effects are mutually coupled, leading to amplification effect that substantially surpasses the individual effect in the degradation of rechargeable batteries.

Experimentally, we integrate in situ transmission electron microscopy (TEM), in situ X-ray diffraction (XRD) and modeling to demonstrate such a coupling effect on the intragranular cracking of a Ni-rich NMC622 layered cathode. We show that in situ heating of delithiated NMC622 particles, even at the first delithiation from the pristine state, leads to explosive nucleation and propagation of intragranular cracking. Such a phenomenon is absent in fully discharged particles. Combining ex situ and in situ TEM observations with chemomechanical modeling, we revealed that in response to the temperature raise, electrochemically induced phase inhomogeneity and oxygen evolution cause, respectively, thermal stress and internal pressure, which constitute the two primary driving forces for the intragranular cracking. The present work demonstrates that the intimate coupling of the electrochemical, thermal, and mechanical processes leads to a much severer effect on the degradation of the cathode [1].

In order to observe the initiation of crack propagation and revealing the underlying atomistic mechanism, we conducted in situ HRTEM observations on a crack tip to directly monitor the lattice evolution during heating. We noticed that when the temperature reached $150{ }^{\circ} \mathrm{C}$, the crack contrast became brighter and the cracking gap wider. HRTEM imaging confirmed that the crack center was transformed into the rock salt phase, while the layered structure remained in the crack-free regions. The phase transformation indicates poor thermal stability at the crack surface and heating induced thermal decomposition first occurs at cracked regions. From room temperature to $150{ }^{\circ} \mathrm{C}$, the crack propagated a few nanometers. Further heating the sample to $172{ }^{\circ} \mathrm{C}$, the crack propagated further. Above $172{ }^{\circ} \mathrm{C}$, the crack tip arrested, suggesting that the thermally induced driving force for crack propagation no longer exceeds the fracture resistance. As the first step, the crack surface region is locally decomposed into rock salt structure, accompanied with the release of oxygen molecules (Figure 1).

In situ STEM observation reveals that the response of delithiated NMC622 to heat treatment resembles the electrochemically cycling induced battery performance decay, featuring nucleation and propagation of intragranular cracking. In-situ HRTEM characterizations evidence that heating induced intragranular cracking is accompanied with surface phase transformation and oxygen gas release. Comprehensive 
mechanics analyses and modeling manifest that the thermal stress due to material phase heterogeneity and internal pressure due to oxygen release constitute two major driving forces for intergranular cracking. Taken together, the observed intragranular cracking resembles the popcorn fracture mechanism under heating. Our studies offer insights into how the electrochemical process triggers thermal and mechanical processes in battery operation, and how these effects are intimately coupled and mutually strengthened in the degradation of layered cathode materials. The fundamental understanding also shed light on the mitigation of the degradation of battery materials under complicated electrochemical and thermomechanical conditions [2].

\section{References:}

[1] P Yan et al., Nature Communications 9 (2018), p. 2437.

[2] The work was conducted in the William R. Wiley Environmental Molecular Sciences Laboratory (EMSL), a national scientific user facility sponsored by DOE's Office of Biological and Environmental Research and located at PNNL.

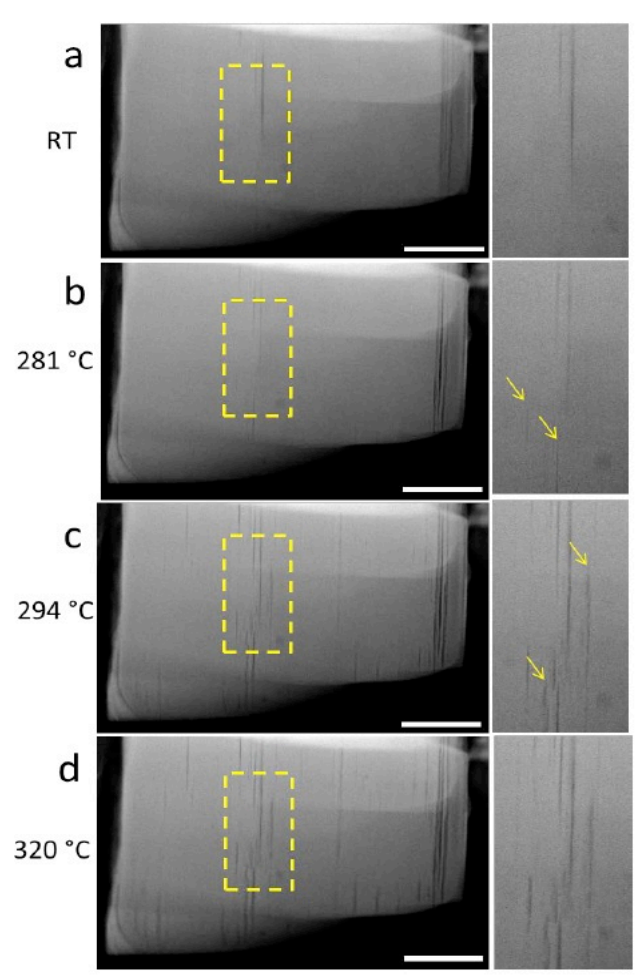

Figure 1. Snapshots during in situ heating. A delithiated $\mathrm{LiNi}_{0.6} \mathrm{Mn}_{0.2} \mathrm{Co}_{0.2} \mathrm{O}_{2}$ particle (charged to $4.7 \mathrm{~V}$ vs Li metal) is heated from room temperature (a) to $281{ }^{\circ} \mathrm{C}(\mathbf{b}), 294{ }^{\circ} \mathrm{C}(\mathbf{c})$, and $320^{\circ} \mathrm{C}(\mathbf{d})$. Yellow arrows indicate new cracks. The scale bars are $200 \mathrm{~nm}$. 